\title{
A computational study of some electric and magnetic properties of gaseous $\mathrm{BF}_{3}$ and $\mathrm{BCl}_{3}$
}

\author{
Antonio Rizzo a) and Chiara Cappellib) \\ Istituto per i Processi Chimico-Fisici del Consiglio Nazionale delle Ricerche, via G. Moruzzi 1, I-56124 \\ Pisa, Italy \\ José Miguel Junquera-Hernández, \\ Alfredo M. J. Sánchez de Merás, and José Sánchez-Marín \\ Departamento de Química Física, Instituto de Ciencia Molecular (ICMol), Universidad de Valencia, \\ Doctor Moliner, 5046100 Burjassot (Valencia), Spain
}

David J. D. Wilson and Trygve Helgaker

Department of Chemistry, University of Oslo, P.O. Box 1033 Blindern, N-0315 Oslo, Norway

(Received 10 June 2005; accepted 20 July 2005; published online 21 September 2005)

\begin{abstract}
We present the results of an extended computational study of the electric and magnetic properties connected to Cotton-Mouton birefringences, on the trifluoro- and trichloroborides in the gas phase. The electric dipole polarizabilities, magnetizabilities, quadrupole moments, and higher-order hypersusceptibilities-expressed as quadratic and cubic frequency-dependent response functionsare computed within Hartree-Fock, density-functional, and coupled-cluster response theories employing singly and doubly augmented correlation-consistent basis sets and London orbitals in the magnetic property calculations. The results, which illustrate the capability of time-dependent density-functional theory for electron-rich systems, are compared with available experimental data. Revised values of both experimentally derived quadrupole moment of $\mathrm{BF}_{3}, 2.72 \pm 0.15$ a.u., and magnetizability anisotropy of $\mathrm{BCl}_{3},-0.45 \pm 0.09$ a.u., both obtained in birefringence experiments that neglect the effects of higher-order hypersusceptibilities, are presented. In the theoretical limit the traceless quadrupole moments of $\mathrm{BF}_{3}$ and $\mathrm{BCl}_{3}$ are determined to be $3.00 \pm 0.01$ and $0.71 \pm 0.01$ a.u., respectively. (C) 2005 American Institute of Physics. [DOI: 10.1063/1.2034487]
\end{abstract}

\section{INTRODUCTION}

The study of the magnetic and electric properties of the boron trihalides $\mathrm{B} X_{3}(X=\mathrm{F}, \mathrm{Cl}, \mathrm{Br}$, and $\mathrm{I})$ has proven a difficult proposition for experimentalists and theoreticians alike, since these systems are corrosive, highly reactive, and electron rich. Nevertheless, there is an obvious interest for chemists in the molecular properties of such species: the $\mathrm{B} X_{3}$ molecules are among the few stable $X Y_{3}$ gases of $D_{3 h}$ symmetry $;^{1}$ the fluorides and chlorides of boron are commonly used in manufacturing, ${ }^{2,3}$ while the Lewis acidity of the boron trihalides is also well known and studied. ${ }^{4-8}$

The electronic charge structure of these simple molecules has been the subject of recent experimental studies, typically from linear birefringence measurements. ${ }^{9-14}$ A linear birefringence is the difference in the refractive index along two axes perpendicular to each other and to the direction of propagation of linearly polarized light traversing a sample. It is usually induced by applying static electric and/or magnetic fields with components perpendicular to the direction of propagation. Birefringences constitute a sensitive probe of the response of matter to an external electromagnetic perturbation and moreover offer an attractive route

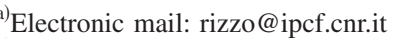

${ }^{b)}$ Present address: Polylab, INFM, Pisa, Italy.
}

to the determination of some important molecular properties such as magnetizabilities, quadrupole moments, polarizabilities, and hyperpolarizabilities. ${ }^{15}$

Two such birefringences are the Cotton-Mouton effect $^{16,17}$ (CME) and the Buckingham effect (BE). ${ }^{18}$ In the $\mathrm{CME}$, the birefringence results from an applied magnetic field, whereas the $\mathrm{BE}$ is observed when an electric-field gradient (EFG) interacts with the beam and the sample. During the last two decades, there has been a growing interest in the study of birefringences both experimentally and theoretically, see Refs. 15, 19, and 20. Recent work by some of the present authors on the birefringences of benzene ${ }^{21}$ and hexafluorobenzene ${ }^{22}$ has illustrated the usefulness of such a theoretical approach for electron-rich systems.

Among the boron trihalides, $\mathrm{BCl}_{3}\left(\mathrm{bp} 12{ }^{\circ} \mathrm{C}\right)$ is the optimal choice for the measurement of birefringences in the vapor phase in comparison with $\mathrm{BF}_{3}\left(\mathrm{bp}-100{ }^{\circ} \mathrm{C}\right)$ and $\mathrm{BBr}_{3}$ (bp $91{ }^{\circ} \mathrm{C}$ ), considering the usual range of temperatures of a birefringence measurement. Indeed, this was one of the reasons why Ritchie and co-workers chose, among the boron trihalides, to measure the $\mathrm{CME}$ of $\mathrm{BCl}_{3}{ }^{9-11,13}$ Still, because of the smallness of the effect in $\mathrm{BCl}_{3}$ and difficulties in measuring over a broad temperature range, it became necessary to ignore the contribution from temperature-independent terms. In an effort to separate the paramagnetic and diamagnetic contributions to the magnetizability anisotropy of $\mathrm{BCl}_{3}$, the lack of an accurate experimental molecular quadrupole 
moment $\Theta$ forced Lamb and Ritchie to use a theoretical value, calculated at the second-order Møller-Plesset (MP2) level of theory in a rather modest Huz-SV basis $(\Theta$ $=1.18$ a.u.). ${ }^{9,12}$

Recently, the accurate determination of the quadrupole moment of $\mathrm{BCl}_{3}$ has been debated. Gierszal et al. reported measurements from a foreign-gas microwave pressure broadening study, yielding a value of $1.52 \pm 0.07$ a.u. ${ }^{12}$ However, questions regarding this work have been raised, ${ }^{13}$ highlighting deficiencies in the experimental technique as well as limitations in the approximations made by Gierszal et al. The experimentally derived value assumed both a vanishing octupole moment of $\mathrm{BCl}_{3}$ and a vanishing quadrupole moment of $\mathrm{CHF}_{3}$, neither of which are valid assumptions. ${ }^{23}$ Clearly, the definitive value of this property has yet to be established.

There are no reported measurements of the $\mathrm{CME}$ of $\mathrm{BF}_{3}$, but Lamb and Ritchie suggested that such an effect can be measured under high pressure. ${ }^{10,11}$ The $\mathrm{BE}$ of $\mathrm{BF}_{3}$ has recently been measured, ${ }^{24}$ whereby the quadrupole moment was determined to be $2.81 \pm 0.16 \mathrm{a} . \mathrm{u}$. The procedure adopted, involving measurements at a single (room) temperature, relies on the neglect of the temperature-independent hyperpolarizability contribution $b(\omega)$. In a number of cases, this approximation has been proven unjustified, see Refs. 15 and 24-29. The only previously reported experimental value for the traceless quadrupole moment of $\mathrm{BF}_{3}$ is 2.50 a.u., obtained from estimates of dipole moments in van der Waals complexes of $\mathrm{BF}_{3}$ with $\mathrm{Ar}, \mathrm{CO}$, and $\mathrm{N}_{2} \cdot{ }^{30}$ Keir and Ritchie also performed MP2/Huz-SV calculations for the quadrupole moment of $\mathrm{BF}_{3}$, yielding a value of 3.21 a.u. ${ }^{9}$

Theoretical studies of the electric and magnetic properties of these interesting molecules are thus timely. The Zeeman properties - the magnetizability, the $g$ tensor, and the quadrupole moment - of the entire series of boron trihalides have recently been the subject of an $a b$ initio study by the authors, employing Hartree-Fock theory and densityfunctional theory (DFT), aimed at discerning trends across the series. ${ }^{31}$ In this parallel work, we carry out a thorough and detailed computational investigation of the properties related to the $\mathrm{CME}$ and $\mathrm{BE}$ of $\mathrm{BF}_{3}$ and $\mathrm{BCl}_{3}$, including other density functionals in the calculation of magnetizability and molecular quadrupole moment than in Ref. 31. More importantly, we perform an accurate coupled-cluster study. Additionally, we examine the electric dipole polarizability and higher-order response functions (hyperpolarizabilities) entering the expressions of the two linear birefringences considered.

The challenges presented to theory by these perhalogenated, electron-rich systems are worth emphasizing. For example, difficulties in calculating the magnetic properties of fluorine containing molecules are well known. ${ }^{32-36}$ Ruud et al. have demonstrated that the additivity of magnetizabilities (known as Pascal's rule) breaks down for fluorine-containing species. $^{34,35}$ For calculations to match the accuracy of the experiment, extensive basis sets are required. Most significantly, the gauge dependence of magnetic properties must be taken into account. ${ }^{35}$ The favored scheme to account for the gauge origin is nowadays the use of London orbitals, also known as gauge-invariant atomic orbitals (GIAOs), which in most cases also improves basis-set convergence. The calculation of DFT magnetizabilities with London orbitals has recently been implemented by the authors ${ }^{37}$ in the DALTON package. $^{38}$

Studies of $\mathrm{C}_{6} \mathrm{H}_{6}$ and $\mathrm{C}_{6} \mathrm{~F}_{6}$ have highlighted the effect that perhalogenation has on the magnetic and electric properties for electron-rich systems. ${ }^{39,40}$ For example, Ritchie and Vrbancich found the magnetizability anisotropy of $\mathrm{C}_{6} \mathrm{~F}_{6}$ to be far larger and the temperature dependence of the CottonMouton and Kerr birefringences to be far more significant than may be expected from an understanding of the molecular properties of benzene. ${ }^{39}$ In a Hartree-Fock study of the quadrupole moment of the fluoro- and chlorobenzenes, Hernández-Trujillo and Vela found that, whereas the quadrupole moment of $\mathrm{C}_{6} \mathrm{H}_{6}$ is converged to within 1 a.u. (15\%) in a small double-zeta basis, successive halogenation reduced the basis-set convergence to the point where it is no longer apparent that the Hartree-Fock quadrupole moment is converged to the same 1-a.u. accuracy (again 15\%) even in a triple-zeta basis. ${ }^{40}$ Since successive fluorination distorts the electron density, we also expect the response of the density to applied electric and magnetic fields and to electric-field gradients to be more complicated compared with the nonhalogenated system.

In view of the challenges that these systems present to theory, we have investigated a number of methods and basis sets for all properties. In particular, the popularity and general usefulness of DFT make it interesting to assess its performance for higher-order properties. Since only a few experimental results of limited accuracy are available, we have also performed high-level ab-initio coupled-cluster singles and doubles ${ }^{41}$ (CCSD) calculations. In particular, for the molecular quadrupole moment, whose accurate determination is a primary aim of our work, we have carried out extensive coupled-cluster calculations with basis-set extrapolation to determine a theoretical limit for this property.

The paper is organized as follows: in Sec. II, we outline the essential definitions and introduce the relevant molecular properties and their link to time-dependent response theory; in Sec. III, computational details are given; the results are presented and discussed in Sec. IV. Some concluding remarks are collected in Sec. V.

\section{THEORY}

The Cotton-Mouton effect is the birefringence induced on linearly polarized light by an applied external magnetic field. ${ }^{16,17}$ It is the magnetic equivalent of the electric-fieldinduced Kerr birefringence. ${ }^{42,43}$ For the CME, the anisotropy of the refractive index $\Delta n(\lambda, T)=n_{\|}-n_{\perp}$ at wavelength $\lambda$ (frequency $\omega$ ) and temperature $T$ is connected to the microscopic molecular properties of the sample through the molecular function labeled as the "Cotton-Mouton constant" ${ }_{m} C(\lambda, T),{ }^{19,44}$ which, for diamagnetic axial molecules in the limit of an ideal gas at fixed pressure, can be written as

$$
\Delta n(\lambda, T)=\frac{27 B^{2}}{2 V_{m}\left(4 \pi \epsilon_{0}\right)}{ }_{m} C(\lambda, T),
$$




$$
{ }_{m} C(\lambda, T)=\frac{2 \pi N_{A}}{27}\left[\Delta \eta(\omega)+\frac{2}{15 k T} \alpha_{\text {ani }}(\omega) \xi_{\text {ani }}\right],
$$

where $k$ is the Boltzmann constant, $N_{A}$ is Avogadro's number, $\epsilon_{0}$ the vacuum permittivity, $V_{m}$ the molar volume, and $B$ the intensity of the magnetic field. Equation (2) introduces the anisotropies of the frequency-dependent electric dipole polarizability ( $z$ axis along the principal molecular axis)

$$
\alpha_{\text {ani }}(\omega)=\alpha_{z z}(\omega)-\alpha_{x z z}(\omega),
$$

of the magnetizability

$$
\xi_{\text {ani }}=\xi_{z z}-\xi_{x x}
$$

and of the frequency-dependent hypermagnetizability

$$
\Delta \eta(\omega)=\frac{1}{5}\left[\eta_{\alpha \beta, \alpha \beta}(\omega)-\frac{1}{3} \eta_{\alpha \alpha, \beta \beta}(\omega)\right] .
$$

For comparison with experimental observations, we use a definition of $\Delta n(\lambda, T)$ that corresponds to the anisotropy observed for a magnetic induction of $1 \mathrm{~T}$ and a pressure of 1 atm, indicated as $\Delta n_{u}{ }^{19}$

In the $\mathrm{BE}$, the birefringence is observed when linearly polarized light traverses the sample in a direction perpendicular to an applied external electric-field gradient $\nabla E .^{18,25}$ With $\nabla E$ arranged so that $\nabla E=\nabla E_{x x}=-\nabla E_{y y}$ and $\nabla E_{z z}=0$, the anisotropy in the refractive index is observed as $\Delta n(\lambda, T)=n_{x}-n_{y}$. The "Buckingham constant" ${ }_{m} Q(\lambda, T)$ connects the anisotropy of the refractive index to the strength of the EFG and to the microscopic properties of the gas. ${ }^{45-47}$ For an axial molecule,

$$
\begin{aligned}
\Delta n(\lambda, T) & =\frac{3 \nabla E}{2 V_{m}}{ }_{m} Q(\lambda, T), \\
{ }_{m} Q(\lambda, T) & =\frac{N_{A}}{3 \epsilon_{0}}\left[b(\omega)+\frac{2}{15 k T} \alpha_{\text {ani }}(\omega) \Theta\right],
\end{aligned}
$$

where $\Theta$ is the symmetry unique traceless permanent electric-quadrupole moment $\left(\Theta_{z z}\right)$ and

$$
b(\omega)=\frac{2}{15} B_{\alpha \beta, \alpha \beta}-\frac{2}{15} \mathcal{B}_{\alpha, \alpha \beta, \beta}-\frac{2}{3 \omega} \varepsilon_{\alpha \beta \gamma} J_{\alpha, \beta, \gamma}^{\prime}
$$

includes tensor components of the electric dipole-electric dipole-electric quadrupole hyperpolarizability $B$, the electric dipole-electric quadrupole-electric dipole hyperpolarizability $\mathcal{B}$, and the electric dipole-magnetic dipole-electric dipole hyperpolarizability $J^{\prime}$. The symbol $\varepsilon_{\alpha \beta \gamma}$ represents the LeviCivita alternating tensor.

In Eqs. (2) and (7), the quantities within the square brackets are separated into a temperature-independent contribution involving higher-order frequency-dependent response properties $\Delta \eta(\omega)$ and $b(\omega)$, respectively, and a temperaturedependent contribution. For theoretical calculations, it is practical to express all these properties in terms of response functions ${ }^{19,27,48,49}$

$$
\begin{aligned}
& \alpha_{\alpha \beta}(\omega)=-\left\langle\left\langle\mu_{\alpha} ; \mu_{\beta}\right\rangle\right\rangle_{\omega}, \\
& \eta_{\alpha \beta, \gamma \delta}(\omega)=\eta_{\alpha \beta, \gamma \delta}^{\mathrm{para}}(\omega)+\eta_{\alpha \beta, \gamma \delta}^{\mathrm{dia}}(\omega),
\end{aligned}
$$

$$
\begin{aligned}
& \eta_{\alpha \beta, \gamma \delta}^{\mathrm{para}}(\omega)=-\left\langle\left\langle\mu_{\alpha} ; \mu_{\beta}, m_{\gamma}, m_{\delta}\right\rangle\right\rangle_{\omega, 0,0}, \\
& \eta_{\alpha \beta, \gamma \delta}^{\mathrm{dia}}(\omega)=-\left\langle\left\langle\mu_{\alpha} ; \mu_{\beta}, \xi_{\gamma \delta}^{\mathrm{dia}}\right\rangle\right\rangle_{\omega, 0}, \\
& B_{\alpha \beta, \gamma \delta}(-\omega ; \omega, 0)=\left\langle\left\langle\mu_{\alpha} ; \mu_{\beta}, \Theta_{\gamma \delta}\right\rangle\right\rangle_{\omega, 0}, \\
& \mathcal{B}_{\alpha, \beta \gamma, \delta}(-\omega ; \omega, 0)=\left\langle\left\langle\mu_{\alpha} ; \Theta_{\beta \gamma}, \mu_{\delta}\right\rangle\right\rangle_{\omega, 0}, \\
& J_{\alpha, \beta, \gamma}^{\prime}(-\omega ; \omega, 0)=i\left\langle\left\langle\mu_{\alpha} ; m_{\beta}, \mu_{\gamma}\right\rangle\right\rangle_{\omega, 0} .
\end{aligned}
$$

Here the superscripts para and dia distinguish the paramagnetic and diamagnetic contributions (see Ref. 19 for a review of the subject), $\mu_{\alpha}$ is the electric dipole operator, $m_{\alpha}$ is the magnetic dipole operator, $\xi_{\alpha \beta}^{\mathrm{dia}}$ is the diamagnetic susceptibility operator, and $\Theta_{\alpha \beta}$ is the traceless electric quadrupole moment operator.

\section{COMPUTATIONAL DETAILS}

Experimental $D_{3 h}$ equilibrium geometries were employed in all calculations, with $\mathrm{BF}$ and $\mathrm{BCl}$ bond lengths of $130.70 \mathrm{pm}$ (Ref. 50) and $174.21 \mathrm{pm},{ }^{51}$ respectively. With the exception of some of the higher-order electric moments, all calculations were performed with a locally modified version of the DALTON program ${ }^{38}$ that includes DFT modules up to cubic response. ${ }^{52,53}$ The octupole-moment and MP2 quadrupole-moment calculations were performed within GAUSSIAN. ${ }^{54}$

All properties were calculated with the Hartree-Fock, DFT, and CCSD methods. Three density functionals were considered: the local-density approximation (LDA), ${ }^{55}$ Becke3-parameter-Lee-Yang-Parr (B3LYP) ${ }^{56}$ and Keal-Tozer KT1 (Ref. 57) functionals. Although the latter has been shown to be particularly accurate in the calculation of molecular magnetic properties, ${ }^{37,57}$ its performance for electric properties has not been thoroughly investigated. ${ }^{58}$ It is therefore of some interest to compare the KT1 functional with the LDA and B3LYP functionals for mixed magnetic and electric properties. The B3LYP results for the magnetizability tensor and quadrupole moments of $\mathrm{BF}_{3}$ and $\mathrm{BCl}_{3}$ were presented in Ref. 31, together with Hartree-Fock and KT2 functional ${ }^{59}$ results.

The singly and doubly augmented correlation-consistent basis sets of Dunning ${ }^{60}$ were employed for all calculations. For all higher-order response properties, we considered the aug-cc-pVXZ and daug-cc-pVXZ (where $X=D, \mathrm{~T}$ ) basis sets. For $\mathrm{BF}_{3}$, we also carried out aug-cc-pVQZ calculations of all properties. Convergence to the basis-set limit for the quadrupole moments of $\mathrm{BF}_{3}$ and $\mathrm{BCl}_{3}$ was investigated for the Hartree-Fock, DFT, MP2, and coupled-cluster models.

As indicated above, the CCSD, CCSD with a perturbative triples correction $[\operatorname{CCSD}(\mathrm{T})]$, and coupled-cluster-triples $^{61}[\mathrm{CC}(3)]$ calculations were carried out with DALTON. ${ }^{38}$ The CCSD implementation in DALTON, including response-function modules, is described in Refs. 62-64. At the CCSD level, traceless quadrupole moments were calculated by direct contraction of the corresponding density matrix with the appropriate integrals ${ }^{63}$ and without orbital relaxation effects, which are small. To extrapolate to the basis-set limit for the coupled-cluster and MP2 methods, 
TABLE I. $\mathrm{BF}_{3}$. Electric dipole polarizability at $\lambda=632.8 \mathrm{~nm}$ and molecular magnetizability anisotropy (in a.u.).

\begin{tabular}{|c|c|c|c|c|c|c|}
\hline Wave function & Basis & $\alpha_{x: x}(\omega)$ & $\alpha_{z z}(\omega)$ & $\alpha_{\mathrm{iso}}(\omega)$ & $\alpha_{\mathrm{ani}}(\omega)$ & $\xi_{\mathrm{ani}}$ \\
\hline \multirow[t]{5}{*}{$\mathrm{HF}^{\mathrm{a}}$} & aug-cc-pVDZ & 14.32 & 11.32 & 13.32 & -2.99 & -0.37 \\
\hline & daug-cc-pVDZ & 14.74 & 11.73 & 13.74 & -3.01 & -0.22 \\
\hline & aug-cc-pVTZ & 14.63 & 11.77 & 13.68 & -2.86 & -0.33 \\
\hline & daug-cc-pVTZ & 14.69 & 11.90 & 13.76 & -2.78 & -0.32 \\
\hline & aug-cc-pVQZ & 14.66 & 11.88 & 13.74 & 2.79 & -0.33 \\
\hline \multirow[t]{5}{*}{ LDA-DFT } & aug-cc-pVDZ & 18.34 & 13.84 & 16.84 & -4.49 & -0.53 \\
\hline & daug-cc-pVDZ & 18.82 & 14.40 & 17.35 & -4.42 & -0.47 \\
\hline & aug-cc-pVTZ & 18.69 & 14.37 & 17.25 & -4.33 & -0.50 \\
\hline & daug-cc-pVTZ & 18.79 & 14.59 & 17.39 & -4.20 & -0.49 \\
\hline & aug-cc-pVQZ & 18.74 & 14.52 & 17.33 & -4.21 & -0.50 \\
\hline \multirow[t]{5}{*}{ B3LYP-DFT } & aug-cc-pVDZ & 17.27 & 13.20 & 15.91 & -4.07 & -0.46 \\
\hline & daug-cc-pVDZ & 17.72 & 13.72 & 16.38 & -4.00 & -0.39 \\
\hline & aug-cc-pVTZ & 17.57 & 13.68 & 16.27 & -3.88 & -0.43 \\
\hline & daug-cc-pVTZ & 17.66 & 13.89 & 16.40 & -3.77 & -0.42 \\
\hline & aug-cc-pVQZ & 17.61 & 13.82 & 16.35 & -3.78 & -0.43 \\
\hline \multirow[t]{5}{*}{ KT1-DFT } & aug-cc-pVDZ & 18.66 & 14.25 & 17.19 & -4.41 & -0.45 \\
\hline & daug-cc-pVDZ & 19.16 & 14.83 & 17.71 & -4.33 & -0.37 \\
\hline & aug-cc-pVTZ & 19.05 & 14.84 & 17.65 & -4.20 & -0.42 \\
\hline & daug-cc-pVTZ & 19.16 & 15.07 & 17.80 & -4.08 & -0.41 \\
\hline & aug-cc-pVQZ & 19.12 & 15.01 & 17.75 & -4.11 & -0.42 \\
\hline \multirow[t]{3}{*}{ CCSD } & aug-cc-pVDz & 16.94 & 12.98 & 15.62 & -3.97 & \\
\hline & daug-cc-pVDZ & 17.46 & 13.54 & 16.16 & -3.93 & \\
\hline & aug-cc-pVTZ & 17.11 & 13.39 & 15.87 & -3.72 & \\
\hline $\mathrm{HF}^{\mathrm{b}}$ & Huz-SV & 13.9 & 10.7 & 12.9 & -3.2 & \\
\hline $\mathrm{MP} 2^{\mathrm{b}}$ & Huz-SV & 16.6 & 12.4 & 15.2 & -4.1 & \\
\hline $\mathrm{HF}(\mathrm{IGLO})^{\mathrm{c}}$ & H-III & & & & & -0.33 \\
\hline \multirow[t]{2}{*}{ Expt. } & & $17.7^{\mathrm{b}}$ & $13.9^{\mathrm{b}}$ & $16.4^{\mathrm{d}}$ & $-3.8 \pm 0.2^{\mathrm{b}}$ & \\
\hline & & & & $22.3^{\mathrm{e}}$ & & \\
\hline
\end{tabular}

${ }^{\mathrm{a}}$ For detailed data on the magnetizability, including tensor components and isotropic average, see Ref. 31.

${ }^{\mathrm{b}}$ From Ref. 9 .

${ }^{c}$ From Ref. 32

${ }^{\mathrm{d}}$ From Ref. 69.

${ }^{\mathrm{e}}$ From Ref. 74.

we used the two-point extrapolation formula ${ }^{65-67}$

$$
P_{\infty}=\frac{P_{X} X^{3}-P_{Y} Y^{3}}{X^{3}-Y^{3}},
$$

where $P_{X}$ and $P_{Y}$ are the property values calculated with the correlation-consistent basis sets (d)aug-cc-pVXZ and (d)aug-cc-pVYZ, respectively. For the Hartree-Fock and DFT methods, we estimated the limit by inspection of the properties calculated with the (d)aug-cc-pVXZ basis sets.

\section{RESULTS AND DISCUSSION}

The results obtained in this study of the birefringences of $\mathrm{BF}_{3}$ and $\mathrm{BCl}_{3}$ are presented and discussed with reference to Tables I-V. We note that a comparison of the calculated properties with experiment is made difficult by the absence of information regarding the effect of molecular vibrations, which in some cases can be large. An example is the electric dipole polarizability of hexafluorobenzene, where the zeropoint vibrational (ZPV) contributions yield an anomalous experimental dispersion, with the static value of the isotropic electric dipole polarizability being larger than at (small) nonvanishing frequencies. ${ }^{68,22}$ In most cases, however, the vibrational corrections are smaller, making a comparison of the purely electronic contributions with the experiment meaningful.

\section{A. The electric dipole polarizability and magnetizability anisotropy}

1. $\mathrm{BF}_{3}$

The calculated frequency-dependent dipole polarizabilities and molecular magnetizability anisotropies of boron trifluoride are listed in Table I. For the elements of the polarizabilities $\alpha_{\alpha \beta}(\omega), \alpha_{\text {iso }}(\omega)$, and $\alpha_{\text {ani }}(\omega)$, the tabulated data indicate that the CCSD/aug-cc-pVTZ results are within 0.1 a.u. of the CCSD basis-set limit. With the aug-cc-pVQZ basis set, the Hartree-Fock and DFT methods both appear to be within 0.15 a.u. of their respective basis-set limits. A comparison of experimental and computational results indicates that the KT1 functional performs less satisfactorily for electric properties than it does for magnetic properties. To exam- 
ine further the suitability of the KTn functionals for electric properties, we have carried out polarizability calculations with the KT3 functional, presumably the best such functional for this property. ${ }^{58}$ However, although the KT3 isotropic and anisotropic values are closer to the experiment than those given by the KT1 functional (KT3/aug-cc-pVQZ values of $\alpha_{\text {iso }}$ and $\alpha_{\text {ani }}$ are 16.89 and -3.99 a.u., respectively), it does not alter our impression that these functionals do not perform as well as B3LYP for electric properties. Note, however, that our comparisons are made without vibrational corrections.

Whereas the Hartree-Fock method yields polarizability components that are significantly smaller in magnitude than the experimental values, ${ }^{9}$ the LDA and KT1 functionals overestimate the polarizability of $\mathrm{BF}_{3}$. The $\mathrm{B} 3 \mathrm{LYP}$ and CCSD models both reproduce experiment within the given error bars, supporting the $\alpha_{\text {iso }}$ estimate of 16.4 a.u. of Keir and Ritchie ${ }^{9}$ rather than the older value of 22.3 a.u. of Watson and Ramaswamy. ${ }^{69}$

For all models, basis-set expansion and augmentation reduce (in absolute value) the polarizability anisotropy. In Table I, we have included two $a b$ initio results reported by Keir and Ritchie. ${ }^{9}$ From an analysis of our data, the basis used by these authors may be considered too small for accurate calculations of this property.

The results obtained for the magnetizability tensor of $\mathrm{BF}_{3}$ with Hartree-Fock theory and the B3LYP and KT2 density functionals were presented and discussed in Ref. 31. Since only the anisotropic magnetizability enters the CottonMouton constant Eq. (2), only this component is listed in Table I. The LDA and KT1 magnetizabilities are within 0.1 a.u. of the previous B3LYP and KT2 results, for the Cartesian as well as isotropic and anisotropic components. ${ }^{31}$

The Hartree-Fock and DFT magnetizabilities of $\mathrm{BF}_{3}$ were obtained employing London orbitals, which are not yet available for coupled-cluster methods in DALTON. ${ }^{38} \mathrm{An}$ approach that is able to cope with the magnetic gauge-origin dependence is mandatory for a reliable treatment of magnetizabilities - non-London Hartree-Fock and CCSD results (not tabulated here) differ significantly from the London results and from the experiment. The London results converge rapidly with basis-set extension, the aug-cc-pVTZ magnetizability components being within 0.01 a.u. of the aug-cc-pVQZ results.

The Hartree-Fock magnetizability anisotropies are consistently more paramagnetic than the DFT values. In our experience, the KT1 and B3LYP functionals are equally well suited to magnetic properties $;{ }^{37}$ their anisotropies here differ by more than 0.01 a.u. Assuming that these functionals give accurate results, we conclude that the Hartree-Fock magnetizability anisotropies are too paramagnetic.

\section{2. $\mathrm{BCl}_{3}$}

The calculated frequency-dependent electric dipole polarizabilities and magnetizability anisotropies of $\mathrm{BCl}_{3}$ are listed in Table II. Except for B3LYP and KT1, adding a second set of diffuse functions and going from a double-zeta to a triple-zeta basis both make the Cartesian components as well as the isotropic and anisotropic components of the polarizability tensor more positive. We estimate that the aug-
cc-pVTZ isotropic and anisotropic polarizabilities are converged to within 1.0 and 0.5 a.u. (2\%), respectively, of the basis-set limit. Compared with the experiment, the HartreeFock method underestimates the in-plane polarizability in the same manner as for $\mathrm{BF}_{3}$, resulting in an underestimation of the isotropic and anisotropic polarizability. The agreement of the CCSD and B3LYP results with the experiment is not as good as for $\mathrm{BF}_{3}$, but the daug-cc-pVTZ results are within twice the reported error bars. The KT1 results are again similar to the LDA results, indicative of the less satisfactory performance of this gradient-corrected functional for electric properties. As for $\mathrm{BF}_{3}$, the KT3/aug-cc-pVTZ isotropic and anisotropic values of 57.72 and -24.25 a.u., respectively, are closer to the experiment than the KT1 values but less accurate than the B3LYP values.

In Table II, we have also included the two results reported by Keir and Ritchie. ${ }^{9}$ As discussed previously, the basis employed by these authors is too small for high accuracy, the Hartree-Fock results, in particular, deviating significantly from the experiment. The agreement of their MP2 result with the experiment is fortuitous, in view of the limitation of the basis set.

As for $\mathrm{BF}_{3}$, the LDA and KT1 magnetizability components (not listed) are very similar to the B3LYP or KT2 values, differing by a few tenths of an a.u. ${ }^{31}$ The Hartree-Fock and DFT magnetizability anisotropies converge rapidly, the aug-cc-pVTZ results being within 0.01 a.u. (1\%) of the basis-set limit. The KT1 and B3LYP magnetizabilities are again similar, the anisotropy differing by less than 0.01 a.u.

In agreement with Lamb and Ritchie, ${ }^{10,11}$ the magnetizability anisotropy of $\mathrm{BF}_{3}$ and $\mathrm{BCl}_{3}$ are both negative, supporting their hypothesis that boron bonds to carbon, oxygen, fluorine, and chlorine are less magnetizable along than across the internuclear axis. The foundation for this hypothesis originates from Pauling, ${ }^{70}$ who recognized the $p \pi-p \pi$ interactions, ionic-covalent resonance, partial double-bond character, and electron delocalization characteristic of the boron bonds, which is surmised to account for the observed negative magnetizability anisotropy. ${ }^{10,11}$ In our previous Zeeman study of the boron trihalides, ${ }^{31}$ the anisotropies of $\mathrm{BBr}_{3}$ and $\mathrm{BI}_{3}$ were found be negative and positive, respectively. Thus, although the hypothesis of Lamb and Ritchie may hold in most cases, at least $\mathrm{BI}_{3}$ is an exception.

\section{B. The quadrupole moment}

The traceless electric quadrupole moments of $\mathrm{BF}_{3}$ and $\mathrm{BCl}_{3}$ have recently been the subject of debate in the literature. ${ }^{9,12,13,24}$ The calculated and experimental quadrupole moments are listed in Table III, with only the daug-cc $-\mathrm{pVXZ}$ results tabulated. Concerning the calculated results, several points are worth making.

First, the Hartree-Fock and DFT quadrupole moments are larger and smaller, respectively, than the coupled-cluster theory results. Second, the poor LDA and KT1 performance for dipole polarizabilities is reflected in the quadrupole moments, with the KT1 functional behaving in a manner similar to the KT2 functional. ${ }^{31}$ As observed in Sec. IV A, although the KT3 functional improves on the KT1 and KT2 results, it 
TABLE II. $\mathrm{BCl}_{3}$. Electric dipole polarizability at $\lambda=632.8 \mathrm{~nm}$ and molecular magnetizability anisotropy (in a.u.).

\begin{tabular}{|c|c|c|c|c|c|c|}
\hline Wave function & Basis & $\alpha_{x x}(\omega)$ & $\alpha_{z z}(\omega)$ & $\alpha_{\text {iso }}(\omega)$ & $\alpha_{\mathrm{ani}}(\omega)$ & $\xi_{\text {ani }}$ \\
\hline \multirow[t]{4}{*}{$\mathrm{HF}^{\mathrm{a}}$} & aug-cc-pVDZ & 57.22 & 38.67 & 51.04 & -18.55 & -0.67 \\
\hline & daug-cc-pVDZ & 58.22 & 40.19 & 52.21 & -18.03 & -0.54 \\
\hline & aug-cc-pVTZ & 58.43 & 40.14 & 52.33 & -18.29 & -0.69 \\
\hline & daug-cc-pVTZ & 58.57 & 40.45 & 52.53 & -18.12 & -0.70 \\
\hline \multirow[t]{4}{*}{ LDA-DFT } & aug-cc-pVDZ & 66.62 & 41.36 & 58.20 & -25.26 & -0.90 \\
\hline & daug-cc-pVDZ & 68.24 & 43.33 & 59.94 & -24.92 & -0.82 \\
\hline & aug-cc-pVTZ & 68.49 & 43.17 & 60.05 & -25.31 & -0.92 \\
\hline & daug-cc-pVTZ & 68.77 & 43.63 & 60.39 & -25.14 & -0.93 \\
\hline \multirow[t]{4}{*}{ B3LYP-DFT ${ }^{\mathrm{a}}$} & aug-cc-pVDZ & 63.84 & 40.48 & 56.06 & -23.36 & -0.80 \\
\hline & daug-cc-pVDZ & 65.31 & 42.38 & 57.67 & -22.94 & -0.71 \\
\hline & aug-cc-pVTZ & 65.43 & 42.22 & 57.69 & -23.21 & -0.79 \\
\hline & daug-cc-pVTZ & 65.69 & 42.71 & 58.03 & -22.99 & -0.81 \\
\hline \multirow[t]{4}{*}{ KT1-DFT } & aug-cc-pVDZ & 67.05 & 41.95 & 58.68 & -25.10 & -0.62 \\
\hline & daug-cc-pVDZ & 68.66 & 43.96 & 60.43 & -24.70 & -0.54 \\
\hline & aug-cc-pVTZ & 68.82 & 43.80 & 60.48 & -25.02 & -0.62 \\
\hline & daug-cc-pVTZ & 69.13 & 44.29 & 60.85 & -24.84 & -0.65 \\
\hline \multirow[t]{3}{*}{ CCSD } & aug-cc-pVDZ & 63.41 & 40.45 & 55.76 & -22.96 & \\
\hline & daug-cc-pVDZ & 64.77 & 42.32 & 57.29 & -22.45 & \\
\hline & aug-cc-pVTZ & 64.39 & 41.98 & 56.92 & -22.41 & \\
\hline $\mathrm{HF}^{\mathrm{b}}$ & Huz-SV & 54.9 & 37.1 & 49.0 & -17.8 & \\
\hline $\mathrm{MP} 2^{\mathrm{b}}$ & Huz-SV & 62.1 & 40.1 & 54.8 & -22.0 & \\
\hline Expt. & & $62.5^{\mathrm{b}}$ & $41.2^{\mathrm{b}}$ & $55.6^{\mathrm{c}}$ & $-21.5 \pm 0.7^{\mathrm{b}}$ & $\begin{array}{l}-0.71 \pm 0.09^{\mathrm{d}} \\
-0.45 \pm 0.09^{\mathrm{e}}\end{array}$ \\
\hline
\end{tabular}

${ }^{\mathrm{a}}$ For detailed data on the magnetizability, including tensor components and isotropic value, see Ref. 31 .

${ }^{\mathrm{b}}$ From Ref. 9.

${ }^{\mathrm{c}}$ From Ref. 75 .

${ }^{\mathrm{d}}$ From Refs. 10 and 11.

${ }^{\mathrm{e}}$ From the data in Refs. 10 and 11 fitted in this work, assuming linear regression with a $T \rightarrow \infty$ value of ${ }_{m} C(\lambda, T)$ equal to our B3LYP-DFT/daug-cc-pVTZ "best value" of $\Delta \eta(\omega)=701.3$ a.u., see Table V.

does not perform quite as well as the B3LYP functional, suggesting that the performance of the KTn functionals for electric properties does not match their performance for magnetic properties. ${ }^{37}$

For all models, basis-set convergence is achieved in the daug-cc-pVTZ basis, whose errors are within 0.010 a.u. of the basis-set limit. We have not listed the singly augmented results but found that the effect of a second set of diffuse functions is small.

Noting that the daug-cc-pVQZ results are close to the basis-set limit, we turn our attention to the coupled-cluster $\mathrm{N}$-electron (excitation-level) convergence. Relative to the CCSD basis-set limit, the CCSD(T) model reduces the quadrupole moment of $\mathrm{BF}_{3}$ by $2.4 \%$ and the $\mathrm{CC}(3)$ model by another $0.1 \%$. In the daug-cc-pVTZ basis, the $\mathrm{CC}(3)$ result is predicted to be within $0.3 \%$ of the basis-set limit. For $\mathrm{BCl}_{3}$, the triples contribution is larger, with a $\operatorname{CCSD}(\mathrm{T})$ reduction of $9.8 \%$ and a further $\mathrm{CC}(3)$ reduction of $0.3 \%$. Assuming a $\mathrm{BCl}_{3}$ basis-set convergence similar to that of $\mathrm{BF}_{3}$, we predict $\operatorname{CCSD}(\mathrm{T})$ and $\mathrm{CC}(3)$ basis-set limits of 0.713 and 0.711 a.u., respectively.

Having established theoretical values for the quadrupole moments of $\mathrm{BF}_{3}$ and $\mathrm{BCl}_{3}$, which we assign to be $3.00 \pm 0.01$ and $0.71 \pm 0.01$ a.u., respectively, we may comment on pre- vious studies of this property. From our basis-set study, it appears that the split-valence results of Ritchie and co-worker ${ }^{9,13}$ are quite far from the MP2 basis-set limit. To illustrate the sensitivity of the quadrupole moment to polarization and diffuse functions in small basis sets, we note that, for the $\mathrm{BF}_{3}$ molecule, MP2 calculations in the $6-31 \mathrm{G}$, $6-31 \mathrm{G}^{*}$, and $6-31+\mathrm{G}^{*}$ basis sets give widely different values of $3.83,2.81$, and 3.20 a.u., respectively. The good agreement between the MP2/Huz-SV and coupled-cluster basis-set limits is therefore fortuitous.

For $\mathrm{BF}_{3}$, an early result of 2.50 a.u., derived from a study of van der Waals complexes, ${ }^{30}$ may be dismissed as too low. A Buckingham birefringence study yields an "apparent" value of $2.81 \pm 0.16 \mathrm{a}$.u., with the temperature-independent $b(\omega)$ contribution neglected. ${ }^{24}$ Including this term in Table III, we obtain a revised value of $2.72 \pm 0.15$ a.u., see details in Sec. IV C 1 below. This inclusion reduces the magnitude of the quadrupole moment of $\mathrm{BF}_{3}$, taking it further away from our best $a b$ initio results. This comparison neglects vibrations, which may in principle account for some of the difference between our best value and the revised experimental value. A preliminary study of the effect of ZPV average on the quadrupole moment of $\mathrm{BF}_{3}$, carried out at Hartree- 
TABLE III. Traceless quadrupole moments of $\mathrm{BF}_{3}$ and $\mathrm{BCl}_{3}$ calculated with daug-cc-pVXZ basis sets (in a.u.).

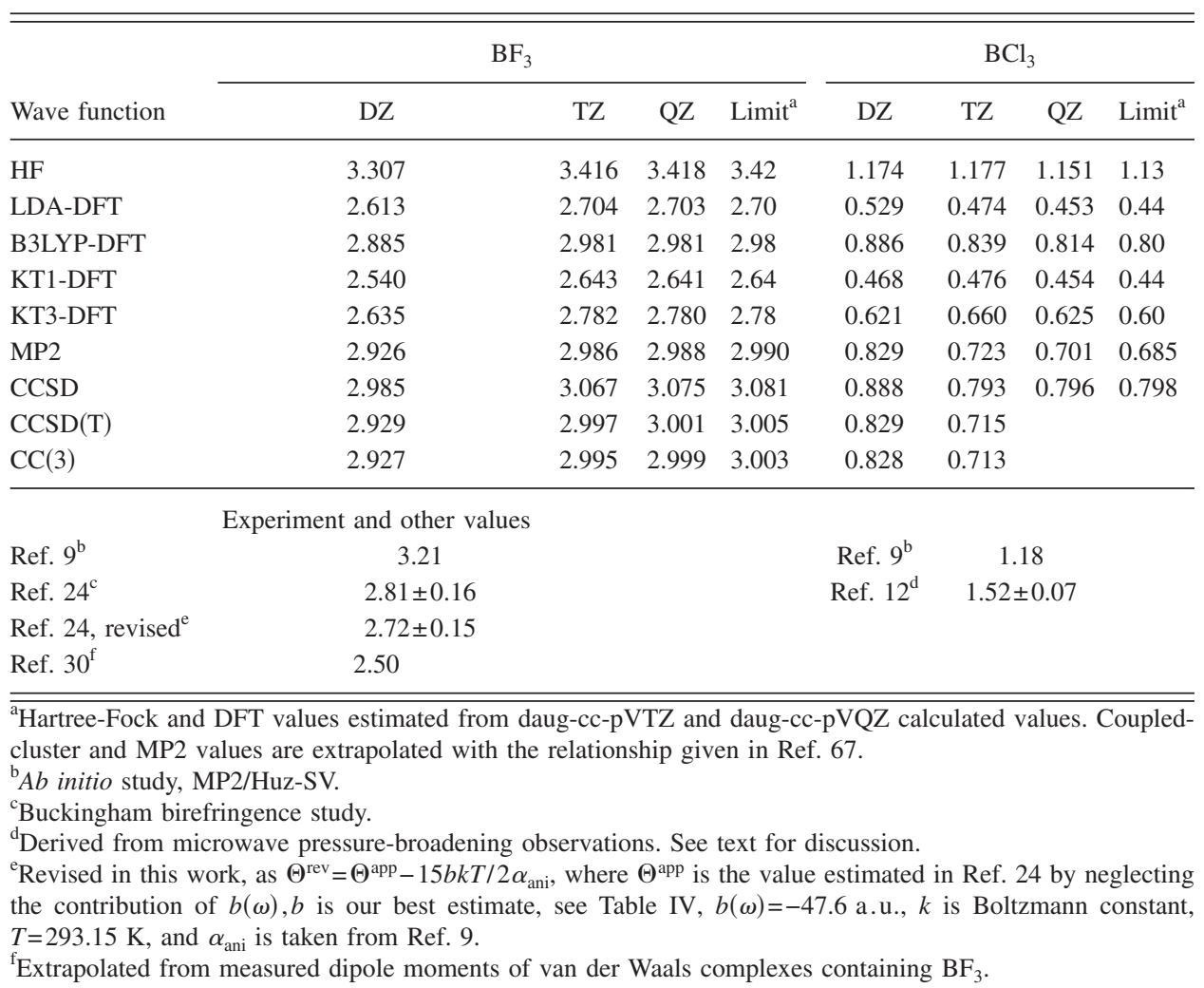

Fock level with the daug-cc-pVTZ basis set, yielded on the other hand a correction on the order of $\approx-0.015$ a.u., rather small although somehow pointing towards the right direction.

As $\mathrm{BF}_{3}$, we do not expect the $\mathrm{BCl}_{3}$ quadrupole moment of Keir and Ritchie ${ }^{9}$ to be close to the MP2 basis-set limit. Indeed, the large differences between their and our $\mathrm{BCl}_{3}$ results support the conclusion that the agreement for $\mathrm{BF}_{3} \mathrm{oc}-$ curs by error cancellation.

The only reported experimental quadrupole moment of $\mathrm{BCl}_{3}$ was derived from a microwave pressure-broadening experiment, with $\mathrm{CHF}_{3}$ as the absorbing species. ${ }^{12}$ However, Ritchie has raised objections to the assumptions made in that work ${ }^{13}$ arguing that the absorber-perturber collision cross section $\sigma$ is not proportional to $\left(\Theta^{2} M\right)^{1 / 3}$ where $M$ is the reduced mass of the absorber-perturber collision pair, and that the observed spectral broadening cannot be interpreted solely on the basis of dipole-quadrupole interactions. The first assumption relied on old $\Theta$ values, for which Ritchie has shown that, when more recent and reliable values are used, the assumed proportionality is qualitatively and quantitatively incorrect. ${ }^{13}$ The second assumption ignores higherorder interactions such as $\mathrm{CHF}_{3}-\mathrm{BCl}_{3}$ quadrupolequadrupole and $\mathrm{CHF}_{3}-\mathrm{BCl}_{3}$ dipole-octupole interactions, which are proportional to $R^{-5}$ and thus shorter ranged than the $R^{-4}$ dipole-quadrupole interactions. Using symmetry arguments, Buckingham demonstrated that $C_{3 v}$ species such as $\mathrm{CHF}_{3}$ possess a nonzero quadrupole moment, whereas $D_{3 h}$ species such as $\mathrm{BCl}_{3}$ exhibit a nonzero molecular octupole moment. $^{23}$

In this work, we investigated the magnitude of these higher-order multipole moments for $\mathrm{BCl}_{3}$. There are two ex- perimental values for the quadrupole moment of $\mathrm{CHF}_{3}$ : the measurement of Meerts and Ozier at 2.79 \pm 0.04 a.u. ${ }^{71}$ and that of Ellenbroek and Dymanus at $2.875 \pm 0.018$ a.u. ${ }^{72}$ Russell has carried out Bruekner-double calculations with a perturbative treatment of triple excitations $[\mathrm{BD}(\mathrm{T})]$, yielding a value of 2.746 a.u. ${ }^{73}$ Our aug-cc-pVQZ values are 2.97 a.u. (Hartree-Fock), 2.40 a.u. (LDA), 2.57 a.u. (B3LYP), and 2.24 a.u. (KT1). The experimental measurements and the $\mathrm{BD}(\mathrm{T})$ value are thus between our Hartree-Fock and B3LYP values, in agreement with the performance of these methods for $\mathrm{BF}_{3}$. Our daug-cc-pVTZ results for the traceless octupole moment of $\mathrm{BCl}_{3}$ are 11.87 a.u. (Hartree-Fock), 11.99 a.u. (B3LYP), and 12.57 a.u. (CCSD).

Our results thus quantitatively support the objections raised by Ritchie $^{9}$ regarding the assumptions in the microwave pressure-broadening experiment ${ }^{12}$ and identify the source of the discrepancy between the experimental values and the results from the current work.

\section{The Cotton-Mouton and Buckingham linear birefringences}

In Tables IV and V, we have collected our results for the hypermagnetizability anisotropy $\Delta \eta(\omega)$, the combination of quadratic responses $b(\omega)$, and the CME, Eq. (2), and Buckingham, Eq. (7), birefringences of $\mathrm{BF}_{3}$ and $\mathrm{BCl}_{3}$, respectively.

\section{1. $\mathrm{BF}_{3}$}

As defined in Sec. II, the Cotton-Mouton and Buckingham constants of $\mathrm{BF}_{3}$ are obtained by combining the electric dipole polarizability anisotropies, the magnetizability 
TABLE IV. $\mathrm{CME}$ and $\mathrm{BE}$ for $\mathrm{BF}_{3}$ at $\lambda=632.8 \mathrm{~nm}$ and $T=273.15 \mathrm{~K}$. a.u., with ${ }_{m} C(\lambda, T)$ in cgs units of $\mathrm{cm}^{3} \mathrm{G}^{-2} \mathrm{~mol}^{-1}\left(4 \pi \epsilon_{0}\right) . \Delta n_{u}$ is the birefringence defined for an induction field $B$ of $1 \mathrm{~T}$ and a pressure $P$ of $1 \mathrm{~atm}$ according to Ref. 19. $\Delta n$ for Buckingham birefringence is given for a pressure of $P=1$ bar and $\mathrm{EFG}$ of $\boldsymbol{\nabla} E=-1 \times 10^{9} \mathrm{~V} \mathrm{~m}^{-2}$

\begin{tabular}{|c|c|c|c|c|c|c|c|}
\hline \multirow[b]{2}{*}{ Wave function } & \multirow[b]{2}{*}{ Basis } & \multirow[b]{2}{*}{$\Delta \eta(\omega)$} & \multirow[b]{2}{*}{${ }_{m} C(\lambda, T) \times 10^{19}$} & CME & \multicolumn{3}{|c|}{ Buckingham } \\
\hline & & & & $\Delta n_{u} \times 10^{14}$ & $b(\omega)$ & ${ }_{m} Q(\lambda, T) \times 10^{-27}$ & $\Delta n \times 10^{15}$ \\
\hline \multirow[t]{5}{*}{$\mathrm{HF}$} & aug-cc-pVDZ & 44.6 & 8.09 & 4.87 & -26.3 & -3.84 & 3.87 \\
\hline & daug-cc-pVDZ & 57.1 & 5.94 & 3.58 & -33.6 & -3.95 & 3.98 \\
\hline & aug-cc-pVTZ & 44.5 & 7.20 & 4.33 & -30.7 & -3.89 & 3.92 \\
\hline & daug-cc-pVTZ & 47.8 & 7.01 & 4.22 & -34.0 & -3.79 & 3.81 \\
\hline & aug-cc-pVQZ & 46.7 & 7.13 & 4.24 & -32.9 & -3.79 & 3.82 \\
\hline \multirow[t]{5}{*}{ LDA-DFT } & aug-cc-pVDZ & 81.9 & 16.85 & 10.12 & -45.7 & -4.51 & 4.54 \\
\hline & daug-cc-pVDZ & 104.8 & 16.06 & 9.64 & -58.2 & -4.64 & 4.67 \\
\hline & aug-cc-pVTZ & 85.9 & 15.85 & 9.52 & -53.3 & -4.71 & 4.74 \\
\hline & daug-cc-pVTZ & 95.0 & 15.60 & 9.37 & -59.8 & -4.56 & 4.60 \\
\hline & aug-cc-pVQZ & 90.6 & 15.69 & 9.43 & -57.1 & -4.58 & 4.61 \\
\hline \multirow[t]{5}{*}{ B3LYP-DFT } & aug-cc-pVDZ & 71.6 & 13.59 & 8.16 & -40.6 & -4.53 & 4.56 \\
\hline & daug-cc-pVDZ & 91.3 & 12.50 & 7.50 & -51.8 & -4.62 & 4.66 \\
\hline & aug-cc-pVTZ & 74.5 & 12.50 & 7.51 & -47.3 & -4.65 & 4.68 \\
\hline & daug-cc-pVTZ & 81.9 & 12.24 & 7.35 & -53.2 & -4.50 & 4.53 \\
\hline & aug-cc-pVQZ & 78.0 & 12.39 & 7.36 & -50.5 & -4.52 & 4.56 \\
\hline \multirow[t]{5}{*}{ KT1-DFT } & aug-cc-pVDZ & 87.0 & 15.01 & 9.01 & -51.4 & -4.33 & 4.36 \\
\hline & daug-cc-pVDZ & 111.1 & 13.53 & 8.12 & -64.5 & -4.44 & 4.47 \\
\hline & aug-cc-pVTZ & 92.5 & 13.61 & 8.17 & -60.0 & -4.49 & 4.52 \\
\hline & daug-cc-pVTZ & 102.4 & 13.56 & 8.14 & -66.9 & -4.37 & 4.40 \\
\hline & aug-cc-pVQZ & 97.7 & 13.58 & 8.15 & -64.0 & -4.39 & 4.42 \\
\hline \multirow[t]{3}{*}{$\operatorname{CCSD}^{\mathrm{a}}$} & aug-cc-pVDZ & 60.5 & 12.89 & 7.66 & -30.0 & -4.58 & 4.62 \\
\hline & daug-cc-pVDZ & 79.5 & 11.87 & 7.06 & -41.9 & -4.56 & 4.59 \\
\hline & aug-cc-pVTZ & 60.1 & 11.54 & 6.86 & -47.6 & -4.68 & 4.71 \\
\hline
\end{tabular}

${ }^{\mathrm{a} B 3 L Y P}$ GIAO magnetizability anisotropies are employed in place of the non-GIAO CCSD results.

anisotropies, and the electric quadrupole moments of Tables I and III with the temperature-independent contribution given in Table IV. The temperature-independent contribution to the CME $\Delta \eta(\omega)$ is significant, constituting more than $20 \%$ of ${ }_{m} C(\lambda, T)$. By contrast, $b(\omega)$ contributes less than $5 \%$ to ${ }_{m} Q(\lambda, T)$.

In the absence of London coupled-cluster calculations, we have used B3LYP magnetizability anisotropies in our $\mathrm{CME}$ studies. For $\mathrm{BF}_{3}$, the anisotropy varies little with the functional-for example, use of the KT1 rather B3LYP anisotropy changes the $\mathrm{CME}$ constant and birefringence by only $3 \%$ in the aug-cc-pVTZ basis.

The higher-order electric properties offer a different challenge to theory than do dipole polarizabilities, as illustrated by the slower basis-set convergence and the larger electron-correlation contribution. Thus, the inclusion of correlation at the DFT level increases $\Delta \eta(\omega)$ and $b(\omega)$ relative to the Hartree-Fock value by at least $60 \%$ and $50 \%$, respectively; sometimes by more than $100 \%$. Note that $\Delta \eta(\omega)$ is obtained, at all levels of theory, without London orbitals, which have not yet been implemented for cubic response functions in DALTON. In these calculations, we used the center of nuclear mass as the gauge origin.

The slower convergence without London orbitals is reflected in the calculated birefringence constants ${ }_{m} C(\lambda, T)$ and ${ }_{m} Q(\lambda, T)$, and in the related birefringences. From our results, we conservatively estimate a Cotton-Mouton constant of ${ }_{m} C(\lambda, T)=(12 \pm 1) \times 10^{-19} \mathrm{~cm}^{3} \mathrm{G}^{-2} \mathrm{~mol}^{-1}\left(4 \pi \epsilon_{0}\right)$ and an associated birefringence of $\Delta n_{u}(\lambda, T)=(7 \pm 1) \times 10^{-14}$, defined for the conditions in Table IV. Similarly, we predict the BE constant to be ${ }_{m} Q(\lambda, T)=(-4.5 \pm 0.3) \times 10^{27}$ a. u., with an associated birefringence of $\Delta n(\lambda, T)=(4.6 \pm 0.3) \times 10^{-15}$.

The CME of $\mathrm{BF}_{3}$ has not yet been measured, but its Buckingham birefringence has been measured by Graham et al. in Ref. 24. These authors report a value of $\Theta$ $+15 b(\omega) k T / 2 \alpha_{\text {ani }}$ of $(12.6 \pm 0.7) \times 10^{-40} \mathrm{C} \mathrm{m}^{2}$ at $20{ }^{\circ} \mathrm{C}$, corresponding, once they assume a vanishing $b(\omega)$ contribution, to $\Theta=2.81 \pm 0.16 \mathrm{a}$.u. Taking a few steps back in their derivation, considering that they assumed $\alpha_{\text {ani }}(\omega)$ at $632.8 \mathrm{~nm}$ to be $(-0.63 \pm 0.03) \times 10^{-40} \mathrm{C}^{2} \mathrm{~m}^{2} \mathrm{~J}^{-1}(-3.8 \pm 0.2$ a.u. $)$ and using for $b(\omega)$ our CCSD/aug-cc-pVTZ result of Table IV (-47.6 a.u.), we obtain the revised experimental quadrupole moment of $\mathrm{BF}_{3}$ of $2.72 \pm 0.15$ a.u. introduced in Sec. IV B. In a similar manner, we can reconstruct a value of ${ }_{m} Q(\lambda, T)$ of $(-3.9 \pm 0.2) \times 10^{27}$ a.u. at $T=293.15 \mathrm{~K}$, as experimentaly observed in Ref. 24, somewhat less negative than predicted $a b$ initio. The discrepancy reflects the difference observed between the $a b$ initio and revised quadrupole moments of boron trifluoride. 
TABLE V. CME and $\mathrm{BE}$ for $\mathrm{BCl}_{3}$ at $\lambda=632.8 \mathrm{~nm}$ and $T=273.15 \mathrm{~K}$. a.u., with ${ }_{m} C(\lambda, T)$ in cgs units of $\mathrm{cm}^{3} \mathrm{G}^{-2} \mathrm{~mol}^{-1}\left(4 \pi \epsilon_{0}\right) . \Delta n_{u}$ is the birefringence defined for an induction field $B$ of $1 \mathrm{~T}$ and a pressure $P$ of $1 \mathrm{~atm}$ according to Ref. 19. $\Delta n$ for Buckingham birefringence is given for a pressure of $P=1$ bar and $\mathrm{EFG}$ of $\boldsymbol{\nabla} E=-1 \times 10^{9} \mathrm{~V} \mathrm{~m}^{-2}$.

\begin{tabular}{|c|c|c|c|c|c|c|c|}
\hline \multirow[b]{2}{*}{ Wave function } & \multirow[b]{2}{*}{ Basis } & \multicolumn{3}{|c|}{ CME } & \multicolumn{3}{|c|}{ Buckingham } \\
\hline & & $\Delta \eta(\omega)$ & ${ }_{m} C(\lambda, T) \times 10^{18}$ & $\Delta n_{u} \times 10^{13}$ & $b(\omega)$ & ${ }_{m} Q(\lambda, T) \times 10^{-27}$ & $\Delta n \times 10^{15}$ \\
\hline \multirow[t]{4}{*}{$\mathrm{HF}$} & aug-cc-pVDZ & 410.6 & 8.78 & 5.29 & -207.4 & -9.509 & 9.58 \\
\hline & daug-cc-pVDZ & 597.4 & 7.82 & 4.71 & -278.9 & -8.931 & 9.00 \\
\hline & aug-cc-pVTZ & 488.7 & 9.14 & 5.50 & -260.2 & -9.221 & 9.29 \\
\hline & daug-cc-pVTZ & 505.0 & 9.21 & 5.55 & -285.0 & -9.008 & 9.07 \\
\hline \multirow[t]{4}{*}{ LDA-DFT } & aug-cc-pVDZ & 547.8 & 15.31 & 9.20 & -247.6 & -6.035 & 6.08 \\
\hline & daug-cc-pVDZ & 874.4 & 15.19 & 9.12 & -344.6 & -5.996 & 6.04 \\
\hline & aug-cc-pVTZ & 707.8 & 16.27 & 9.78 & -311.7 & -5.618 & 5.66 \\
\hline & daug-cc-pVTZ & 775.4 & 16.54 & 9.94 & -352.8 & -5.521 & 5.56 \\
\hline \multirow[t]{4}{*}{ B3LYP-DFT } & aug-cc-pVDZ & 509.5 & 12.72 & 7.65 & -235.7 & -9.002 & 9.07 \\
\hline & daug-cc-pVDZ & 799.6 & 12.45 & 7.47 & -328.1 & -8.733 & 8.80 \\
\hline & aug-cc-pVTZ & 642.8 & 13.13 & 7.89 & -296.3 & -8.592 & 8.65 \\
\hline & daug-cc-pVTZ & 701.3 & 13.34 & 8.01 & -336.2 & -8.341 & 8.40 \\
\hline \multirow[t]{4}{*}{ KT1-DFT } & aug-cc-pVDZ & 571.0 & 11.25 & 6.76 & -261.9 & -5.555 & 5.59 \\
\hline & daug-cc-pVDZ & 905.2 & 11.21 & 6.73 & -360.6 & -5.401 & 5.44 \\
\hline & aug-cc-pVTZ & 739.8 & 11.87 & 7.13 & -325.9 & -5.631 & 5.67 \\
\hline & daug-cc-pVTZ & 812.0 & 12.47 & 7.49 & -369.8 & -5.532 & 5.57 \\
\hline \multirow[t]{3}{*}{$\operatorname{CCSD}^{\mathrm{a}}$} & aug-cc-pVDZ & 446.6 & 12.30 & 7.31 & -227.6 & -9.206 & 9.27 \\
\hline & daug-cc-pVDZ & 692.4 & 11.84 & 7.04 & -310.1 & -8.536 & 8.60 \\
\hline & aug-cc-pVTZ & 547.7 & 12.40 & 7.37 & -284.7 & -7.809 & 7.87 \\
\hline \multicolumn{3}{|c|}{ Extrapolated from experiment } & $8.2 \pm 0.7^{\mathrm{b}}$ & $4.9 \pm 0.7^{\mathrm{b}}$ & & & \\
\hline
\end{tabular}

${ }^{\mathrm{a} B 3 L Y P-D F T}$ GIAO magnetizability anisotropies are employed in place of the non-GIAO CCSD results.

${ }^{b}$ Data in Refs. 10 and 11 fitted in this work, assuming linear regression with a $T \rightarrow \infty$ value of ${ }_{m} C(\lambda, T)$ equal to our B3LYP-DFT/daug-cc-pVTZ "best value" of $\Delta \eta(\omega)=701.3$ a.u.

\section{2. $\mathrm{BCl}_{3}$}

The data employed in the study of the Cotton-Mouton and Buckingham birefringences of $\mathrm{BCl}_{3}$ in Table $\mathrm{V}$ are listed in Tables II and III, combined with the higher-order contribution in Table V. Figure 1 reports the experimental data obtained in the 295.6-361.9-K temperature range by Lamb and Ritchie ${ }^{10,11}$ for the CME of boron trichloride, comparing it with the ab initio results obtained in the largest basis sets.

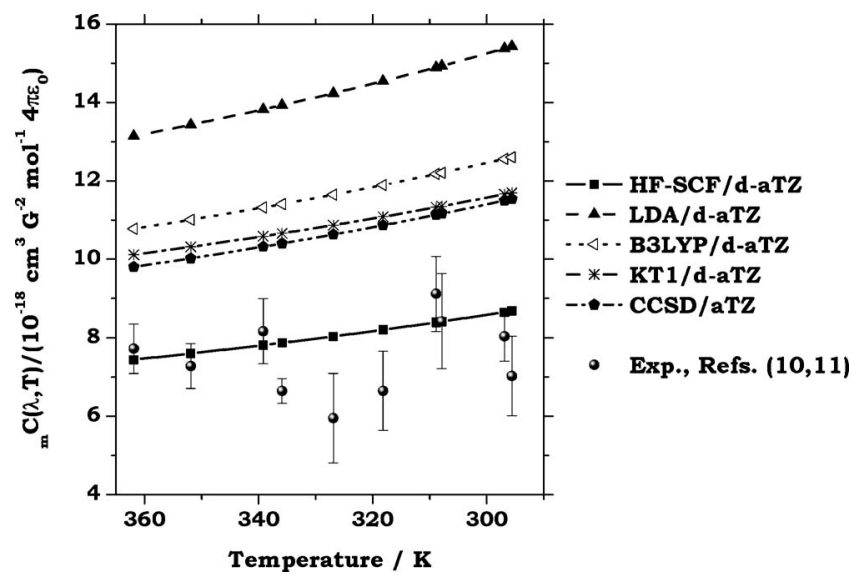

FIG. 1. The temperature dependence of the $\mathrm{CME}$ of $\mathrm{BCl}_{3}$ as computed here, compared to the experiment. The labels "aTZ" and "d-aTZ" stand for augcc-pVTZ and daug-cc-pVTZ, respectively.
As for $\mathrm{BF}_{3}$, the London B3LYP (rather than non-London CCSD) magnetizability anisotropies are used for the CottonMouton constant and for the corresponding birefringence. The magnetizability anisotropy depends more critically on the density functional for $\mathrm{BCl}_{3}$ than for $\mathrm{BF}_{3}$; for example, use of the KT1 rather than B3LYP anisotropies modifies the aug-cc-pVTZ Cotton-Mouton constant by about $20 \%$.

The results presented here allow us to compare the performance of KT1 for electric and magnetic properties. Thus, for the CME constant (which includes magnetic properties via $\xi_{\text {ani }}$ ) and associated birefringence, the KT1 values agree well with the CCSD results and with B3LYP, and they are close to the area where experimental data are distributed, see Fig. 1. Still, it is the Hartree-Fock method that yields results closest to the experiment, whereas LDA is rather off target. By contrast, for the BE, which depends only on the electric properties, the agreement of KT1 with the CCSD (and B3LYP) results is poorer and similar to that of the LDA functional.

The temperature-independent CME contribution is similar to that of $\mathrm{BF}_{3}$, with $\Delta \eta(\omega)$ contributing about $20 \%$ to ${ }_{m} C(\lambda, T)$. By contrast, $b(\omega)$ contributes more than $10 \%$ to the Buckingham constant ${ }_{m} Q(\lambda, T)$ of $\mathrm{BCl}_{3}$, twice the amount of $\mathrm{BF}_{3}$. In their experimental study of the CME, Lamb and Ritchie $^{10,11}$ were unable to identify the temperatureindependent contribution by extrapolation to infinite tem- 
perature because of the smallness of the effect and limited experimental precision, forcing the authors to ignore $\Delta \eta(\omega)$ in the derivation of $\xi_{\text {ani }}$. However, they did suggest that the temperature-independent contribution may easily account for $10 \%$ of the measured value of ${ }_{m} C(\lambda, T)$, increasing the magnitude of the experimentally derived $\left|\xi_{\text {ani }}\right|$. Indeed, our study indicates a temperature-independent contribution of about $20 \%$. In Table V, we have listed an experimental value for the CME of $\mathrm{BCl}_{3}$, but note that this value has been extrapolated to $273.15 \mathrm{~K}$ from a linear regression of the experimental data. ${ }^{10,11}$

In view of the neglected temperature-independent contribution in the paper of Ritchie and Lamb, ${ }^{10,11}$ we have rederived the value for $\xi_{\text {ani }}$. If their experimental data are fitted such that the line, Eq. (2), passes through our estimated intercept (B3LYP/daug-cc-pVTZ, $\Delta \eta=701.3$ a.u.), and if the same value assumed by Lamb and Ritchie is taken for the anisotropy of the electric dipole polarizability $\left(\alpha_{\text {ani }}=\right.$ $-21.5 \pm 0.7),{ }^{9}$ then the experimental estimate for $\xi_{\text {ani }}$ is revised to $-0.45 \pm 0.09$ a.u. We have no estimates of the effects of vibrations on the magnetizability of $\mathrm{BCl}_{3}$. However, some preliminary results for $\mathrm{BF}_{3}$, obtained as for the quadrupole moment, see Sec. IV B, at the Hartree-Fock level and with the daug-cc-pVTZ basis set, indicate that the effect of $\mathrm{ZPV}$ average on the magnetizability anisotropy is in that case of $\approx 5 \%$ the purely electronic property. We have no reason to suspect that ZPV average might play a much stronger role in $\mathrm{BCl}_{3}$. Other sources of error, as, for example, the effect of relativity, are most likely even less important. We therefore conclude that the error bar of 0.09 a.u. given in Ref. 10 for the magnetizability anisotropy of $\mathrm{BCl}_{3}$, and which takes into account also systematic errors, is probably too small.

We predict a Cotton-Mouton constant of ${ }_{m} C(\lambda, T)$ $=(13 \pm 2) \times 10^{-18} \mathrm{~cm}^{3} \mathrm{G}^{-2} \mathrm{~mol}^{-1}\left(4 \pi \epsilon_{0}\right)$ with an associated birefringence of $\Delta n_{u}(\lambda, T)=(7.5 \pm 1.0) \times 10^{-13}$, under the conditions in Table V. With the exception of Hartree-Fock, which displays a surprisingly excellent performance, we are outside the error bars of the experiment, and overestimate the effect. In view of what we have shown above, it is rather unlikely that the causes of discrepancy between our electroncorrelated results and the experiment would be removed by a proper account of the effect of molecular vibrations.

Finally, we predict the BE constant to be ${ }_{m} Q(\lambda, T)$ $=(-8 \pm 1) \times 10^{27} \mathrm{a}$.u. with an associated birefringence of $\Delta n(\lambda, T)=(8 \pm 1) \times 10^{-15}$. No experimental measurements are available for these constants.

\section{CONCLUSION}

We have presented the results of the Hartree-Fock, DFT, and CCSD studies of the electric and magnetic properties of $\mathrm{BF}_{3}$ and $\mathrm{BCl}_{3}$-including the magnetizability anisotropy, quadrupole moment, frequency-dependent electric dipole polarizability, and higher-order response properties-all associated with Cotton-Mouton and Buckingham linear birefringences. Basis-set convergence has been examined and basisset limits established. The performance of DFT is excellent. In particular, the hybrid B3LYP functional gives the best results, with the Keal-Tozer functionals performing less satisfactorily for electric properties than for magnetic properties.

The agreement with the experimental data is satisfactory, particularly in view of the neglect of molecular vibrations. The temperature-independent contribution to the CottonMouton birefringence is about $20 \%$ for both molecules, whereas the contributions to the $\mathrm{BE}$ are about $5 \%$ and $10 \%$ for $\mathrm{BF}_{3}$ and $\mathrm{BCl}_{3}$, respectively.

We have carried a detailed and systematic investigation of the molecular quadrupole moment of both molecules, yielding $3.00 \pm 0.01$ and $0.71 \pm 0.01$ a.u. for $\mathrm{BF}_{3}$ and $\mathrm{BCl}_{3}$, respectively. For $\mathrm{BF}_{3}$, this value is within two standard deviations of our revised experimental measurement, while for $\mathrm{BCl}_{3}$ our value supports the claims of Ritchie ${ }^{10,11,13}$ that the measurement of Gierszal et al. ${ }^{12}$ is inaccurate.

A revision of the linear regression analysis of the CME measurements in $\mathrm{BCl}_{3}$ performed by Lamb and Ritchie ${ }^{10,11}$ yields a revised value of the magnetizability anisotropy of boron trichloride, $\xi_{\text {ani }}=-0.45 \pm 0.09$ a.u., almost $40 \%$ less negative than the original estimate, and in disagreement with our best $a b$ initio results. The Cotton-Mouton constant of $\mathrm{BCl}_{3}$ is indeed overestimated by our electron-correlated approaches in the whole range of temperatures of the original experiment, whereas Hartree-Fock performs excellently. The origin of these discrepancies is apparently not ascribable to the neglect of the effect of molecular vibrations.

\section{ACKNOWLEDGMENTS}

This work has been supported by the European Research and Training Network "Understanding Nanomaterials from a Quantum Perspective" (NANOQUANT), Contract No. MRTN-CT-2003-506842 and by the Norwegian Research Council (Grant No. 154011/420). One of the authors (D.J.D.W.) has been supported by the Norwegian Research Council through a postdoctoral fellowship (Grant No. 155137/432). We also acknowledge a grant of computer time from the Norwegian Supercomputing Program.

${ }^{1}$ Y. Pak and R. C. Woods, J. Chem. Phys. 106, 6424 (1997).

${ }^{2}$ K. K. Baeck and R. J. Bartlett, J. Chem. Phys. 106, 4604 (1997).

${ }^{3}$ C. W. Bauschlicher and A. Ricca, J. Phys. Chem. A 103, 4313 (1999).

${ }^{4}$ H. C. Brown and R. R. Holmes, J. Am. Chem. Soc. 78, 2173 (1956).

${ }^{5}$ F. A. Cotton and G. Wilkinson, Advanced Inorganic Chemistry (Wiley, New York, 1980).

${ }^{6}$ T. Brinck, J. S. Murray, and P. Politzer, Inorg. Chem. 32, 2622 (1993).

${ }^{7}$ V. Jones, G. Frenking, and M. T. Reetz, J. Am. Chem. Soc. 116, 8741 (1994).

${ }^{8}$ S. Fau and G. Frenking, Mol. Phys. 96, 519 (1999).

${ }^{9}$ R. I. Keir and G. L. D. Ritchie, Chem. Phys. Lett. 290, 409 (1998).

${ }^{10}$ D. W. Lamb and G. L. D. Ritchie, Chem. Phys. Lett. 310, 150 (1999).

${ }^{11}$ D. W. Lamb and G. L. D. Ritchie, Chem. Phys. Lett. 317, 653 (2000).

${ }^{12}$ S. Gierszal, J. Galica, and E. Miś-Kuźminńska, Phys. Scr. 67, 525 (2003).

${ }^{13}$ G. L. D. Ritchie, Phys. Scr. 69, 403 (2004).

${ }^{14}$ E. B. Graham and R. E. Raab, Mol. Phys. 52, 1241 (1984).

${ }^{15}$ A. Rizzo and S. Coriani, Adv. Quantum Chem. (in press).

${ }^{16}$ A. Cotton and H. Mouton, C. R. Hebd. Seances Acad. Sci. 141, 317 (1905).

${ }^{17}$ A. Cotton and H. Mouton, C. R. Hebd. Seances Acad. Sci. 141, 349 (1905).

${ }^{18}$ A. D. Buckingham, J. Chem. Phys. 30, 1580 (1959).

${ }^{19}$ C. Rizzo, A. Rizzo, and D. M. Bishop, Int. Rev. Phys. Chem. 16, 81 (1997). 
${ }^{20}$ S. Coriani, A. Halkier, and A. Rizzo, in Recent Research Developments in Chemical Physics, edited by G. Pandalai (Transworld Scientific, Kerala, India, 2001), Vol. 2, p. 1.

${ }^{21}$ A. Rizzo, C. Cappelli, B. Jansík, D. Jonsson, P. Sałek, S. Coriani, and H. Ågren, J. Chem. Phys. 121, 8814 (2004).

${ }^{22}$ A. Rizzo, C. Cappelli, B. Jansík et al., J. Chem. Phys. 122, 234314 (2005).

${ }^{23}$ A. D. Buckingham, in Physical Chemistry, an Advanced Treatise, edited by H. Eyring, D. Henderson, and W. Yost (Academic, New York, 1970), Vol. 4.

${ }^{24}$ C. Graham, D. A. Imrie, and R. E. Raab, Mol. Phys. 93, 49 (1998).

${ }^{25}$ A. D. Buckingham and R. L. Disch, Proc. R. Soc. London, Ser. A 273, 275 (1963).

${ }^{26}$ R. I. Keir, D. W. Lamb, G. L. D. Ritchie, and J. N. Watson, Chem. Phys. Lett. 279, 22 (1997).

${ }^{27}$ S. Coriani, C. Hättig, P. Jørgensen, A. Rizzo, and K. Ruud, J. Chem. Phys. 109, 7176 (1998)

${ }^{28}$ C. Cappelli, U. Ekström, A. Rizzo, and S. Coriani, J. Comput. Methods Sci. Eng. 4, 365 (2004).

${ }^{29}$ J. M. Junquera-Hernández, J. Sánchez-Marín, V. Pérez-Mondéjar, and A. S. de Merás, Chem. Phys. Lett. 378, 211 (2003).

${ }^{30}$ S. E. Novick, J. Phys. Chem. 90, 3871 (1986).

${ }^{31}$ D. J. D. Wilson, T. Helgaker, and A. Rizzo, Mol. Phys. (in press).

${ }^{32}$ U. Fleischer and M. Schindler, Chem. Phys. 120, 103 (1988).

${ }^{33}$ P. O. Åstrand and K. V. Mikkelsen, Magn. Reson. Chem. 36, 92 (1998).

${ }^{34}$ K. Ruud, T. Helgaker, P. Jørgensen, and K. L. Bak, Chem. Phys. Lett. 223, 12 (1994).

${ }^{35}$ K. Ruud, P. O. Åstrand, and P. R. Taylor, J. Phys. Chem. A 105, 9926 (2001).

${ }^{36}$ H. Fukaya and T. Ono, J. Comput. Chem. 25, 51 (2003).

${ }^{37}$ D. J. D. Wilson, C. E. Mohn, and T. Helgaker, J. Chem. Theory Comput. (to be published).

${ }^{38}$ DALTON, a molecular electronic structure program, Release 2.0, 2005; see http://www.kjemi.uio.no/software/dalton/dalton.html

${ }^{39}$ G. L. D. Ritchie and J. Vrbancich, Aust. J. Chem. 35, 869 (1982).

${ }^{40}$ J. Hernández-Trujillo and A. Vela, J. Phys. Chem. 100, 6524 (1996).

${ }^{41}$ G. D. Purvis and R. J. Bartlett, J. Chem. Phys. 76, 1910 (1982).

${ }^{42}$ J. Kerr, Philos. Mag. 50, 337 (1875).

${ }^{43}$ J. Kerr, Philos. Mag. 50, 446 (1875).

${ }^{44}$ A. D. Buckingham and J. A. Pople, Proc. Phys. Soc. London, Sect. B 69 1133 (1956)

${ }^{45}$ A. D. Buckingham and H. C. Longuet-Higgins, Mol. Phys. 14, 63 (1968).

${ }^{46}$ A. D. Buckingham and M. J. Jamieson, Mol. Phys. 22, 117 (1971).

${ }^{47}$ D. A. Imrie and R. E. Raab, Mol. Phys. 74, 833 (1991)

${ }^{48} \mathrm{~J}$. Olsen and P. Jørgensen, in Modern Electronic Structure Theory, edited by D. R. Yarkony (World Scientific, Singapore, 1995), Pt. II, p. 857.

${ }^{49}$ A. Rizzo and S. Coriani, J. Chem. Phys. 119, 11064 (2003).

${ }^{50}$ S. Yamamoto, R. Kuwabara, M. Takami, and K. Kuchitsu, J. Mol. Spectrosc. 115, 333 (1986).

${ }^{51}$ J. H. Callomon, E. Hirota, K. Kuchitsu, W. J. Lafferty, A. G. Maki, and C. S. Pole, in Landolt-Börnstein: Numerical Data and Functional Relationships in Science and Technology, New Series, Group II Atomic and Molecular Physics, edited by K. H. Hellwege and A. M. Hellwege (Springer, New York, 1976), Vol. 7, Chap. 2, p. 15.

${ }^{52}$ B. Jansík, P. Sałek, D. Jonsson, O. Vahtras, and H. Ågren, J. Chem. Phys. 122, 054107 (2005).

${ }^{53}$ B. Jansík, Density functional theory for molecular properties, Ph.D. thesis, KTH Biotechnology, Stockholm, Sweden, 2004.

${ }^{54}$ M. J. Frisch, G. W. Trucks, H. B. Schlegel et al., Gaussian 03, Revision A.1, Gaussian, Inc., Pittsburgh, PA, 2003.

${ }^{55}$ S. H. Vosko, L. Wilk, and M. Nusair, Can. J. Phys. 58, 1200 (1980).

${ }^{56}$ A. D. Becke, J. Chem. Phys. 98, 5648 (1993).

${ }^{57}$ T. W. Keal and D. J. Tozer, J. Chem. Phys. 119, 3015 (2003).

${ }^{58}$ T. W. Keal and D. J. Tozer, J. Chem. Phys. 121, 5654 (2004).

${ }^{59}$ T. W. Keal, D. J. Tozer, and T. Helgaker, Chem. Phys. Lett. 391, 374 (2004).

${ }^{60}$ T. H. Dunning, Jr., J. Chem. Phys. 90, 1007 (1989)

${ }^{61}$ K. Hald and P. Jørgensen, Phys. Chem. Chem. Phys. 4, 5221 (2002).

${ }^{62}$ O. Christiansen, A. Halkier, H. Koch, P. Jørgensen, and T. Helgaker, J. Chem. Phys. 108, 2801 (1998).

${ }^{63}$ A. Halkier, H. Koch, O. Christiansen, P. Jørgensen, and T. Helgaker, J. Chem. Phys. 107, 849 (1997).

${ }^{64}$ C. Hättig, O. Christiansen, H. Koch, and P. Jørgensen, Chem. Phys. Lett. 269, 428 (1997).

${ }^{65}$ T. Helgaker, W. Klopper, H. Koch, and J. Noga, J. Phys. Chem. 106, 9639 (1997).

${ }^{66}$ A. Halkier, T. Helgaker, P. Jørgensen, W. Klopper, H. Koch, J. Olsen, and A. K. Wilson, Chem. Phys. Lett. 286, 243 (1998).

${ }^{67}$ T. Helgaker, P. Jørgensen, and J. Olsen, Molecular Electronic-Structure Theory (Wiley, Chichester, 1999).

${ }^{68}$ G. L. D. Ritchie and J. N. Watson, Chem. Phys. Lett. 322, 143 (2000).

${ }^{69}$ H. E. Watson and K. L. Ramaswamy, Proc. R. Soc. London, Ser. A 156, 144 (1936).

${ }^{70}$ L. Pauling, The Nature of the Chemical Bond, 2nd. ed. (Cornell University Press, Ithaca, NY, 1940).

${ }^{71}$ W. L. Meerts and I. Ozier, J. Chem. Phys. 75, 596 (1981).

${ }^{72}$ A. W. Ellenbroek and A. Dymanus, Chem. Phys. 35, 227 (1978).

${ }^{73}$ A. J. Russell and M. A. Spackman, Mol. Phys. 98, 633 (2000).

${ }^{74}$ A. A. Maryott and F. Buckley, Circular No. 537 (National Bureau of Standards, Washington DC, 1953), p. 11.

${ }^{75}$ R. J. W. LeFèvre, Proc. Chem. Soc., London 1958, 283. 